\section{The CEK1-mediated mitogen-activated protein kinase pathway in the fungal pathogen Candida albicans}

\author{
Elvira Román, Jesús Pla \\ Departamento de Microbiología II, \\ Facultad de Farmacia, Universidad \\ Complutense de Madrid, Spain
}

\section{Abstract}

Mitogen-activated protein kinases (MAPK) mediated signal transduction pathways are essential for the adaptation of living organisms to environmental changes. In pathogenic fungi, these MAPK cascades govern the response to many types of situations, and are essential for the successful establishment of the fungus within the host. Therefore, they influence virulence and can be considered as promising therapeutic targets. In the opportunistic pathogen Candida albicans, the Cek1mediated pathway was identified long time ago as an important virulence determinant in certain animal models. We will review here the recent work that reveals the role that this route plays in three important processes for the cell: osmotic adaptation, fungal morphogenesis and cell wall remodeling. We will also show the complementary (and sometimes opposite) roles that under specific circumstances the high osmolarity glycerol and $C E K 1$ pathways play in $C$. albicans biology, especially in the context of the interaction with the mammalian host.

\section{Introduction}

Almost every living organism is constantly exposed to environmental changes that challenge its survival. Cells have, accordingly, developed different mechanisms that mediate adaptation to these changes. Mitogen-activated protein kinase (MAPK) pathways are biochemical transducers of such environmental alterations. A stimulus, normally sensed by different surface receptors, is transmitted via phosphorylation to a MAPK that becomes translocated to the nucleus of the cell where it activates different targets [normally transcription factors (TF)] that mediate either down or up regulation of the TF target genes, therefore allowing the correct response to the initial stimulus. To ensure the timing and intensity of the response, and due to the fact that some elements are present in more than one cascade, regulatory mechanisms must be present in the cells. These include protein phosphatases (that dephosphorylate MAPKs and other proteins), ${ }^{1}$ docking interactions (ensuring correct and tight protein-protein interactions), ${ }^{2}$ crosstalk inhibition (that ensure the inhibition of one pathway once another is active) and kinetic insulation (that allow different cascades respond to the same stimulus differently in terms of kinetic or threshold).

MAPK-mediated signaling is attractive in the field of fungal pathogenicity. Not only MAPK routes are involved in adaptation to stress but they do also participate in processes like mating or morphogenesis that regulate important aspects of fungal virulence as occurs, for example, in Cryptococcus neoformans. ${ }^{3,4}$ Cells defective in certain elements of MAPK pathways are often attenuated in virulence, being unable to initiate or establish an infection in different mammalian and nonmammalian hosts. 5 Selective inhibition of MAPK pathways is, therefore, a potential attractive way to control infections caused by fungi. This is especially interesting in view of the relatively limited repertoire of antibiotics available to treat fungal infections compared to bacterial ones, a fact that is largely dependent on the close similarities that exist both in the mammalian and fungal cells, both eukaryotic. Furthermore, resistance to some of the most commonly used antifungals (the azole family) is becoming frequent ${ }^{6}$ and may limit in a near future its usefulness.

Among pathogenic fungi, Candida albicans is probably the most extensively known model in biological research. This position is a reflection of the high incidence of the infections caused by this microbe, despite the increase in the diversity of etiological agents causing fungal infections in the last years. ${ }^{7}$ C. albicans is responsible for the $8-10 \%$ of bloodstream nosocomial infections in the USA ${ }^{8}$ and is a serious risk at hospital's intensive care units. This yeast is a normal member of the human microbiota, being encountered as colonizer of the vaginal and gastrointestinal tract in about 30 $70 \%$ of human population. This last location is considered the main reservoir in humans; ${ }^{9}$ upon alterations of the host immune response or mucosal barriers, translocation to the bloodstream occurs and colonization of internal organs like liver, kidneys, spleen or brain takes place, causing extremely severe diseases with mortality that can be as high as $47 \% .10$ Immune deficiencies present in individuals with certain genetic defects (mainly neutrophils or $\mathrm{T}_{\mathrm{H}} 17$ response, undergoing cancer chemotherapy and/or organ transplant and AIDS are important predisposing factors to candidiasis).11-14

Research in C. albicans was long hampered by their biological peculiarities: its diploidy, absence of a sexual cycle similar to $S$. cerevisi$a e$ and lack of natural plasmids, resulted in a
Correspondence: Elvira Román, Departamento de Microbiología II, Facultad de Farmacia, Universidad Complutense de Madrid, Plaza de Ramón y Cajal s/n, E-28040 Madrid, Spain.

Tel. + 34.91.3941617 - Fax: + 34.91.3941745

E-mail: elvirarg@farm.ucm.es

Key words: mitogen-activated protein kinases, Candida albicans, high osmolarity glycerol, STE vegetative growth pathway, crosstalk.

Conflict of interests: the authors report no conflict of interests.

Funding: the work in our lab is supported by Grants BIO2012-31839 and PIM2010EPA-00658.

Received for publication: 2 October 2012.

Revision received: 20 March 2013.

Accepted for publication: 8 April 2013.

This work is licensed under a Creative Commons Attribution NonCommercial 3.0 License (CC BYNC 3.0).

CCCopyright E. Román and J. Pla, 2013

Licensee PAGEPress, Italy

MAP Kinase 2013; 2:e5

doi:10.4081/mk.2013.e5

difficult genetic manipulation. 15,16 However, its genome has been now fully sequenced, 17 its almost sexual cycle (being dependent on a morphogenic switch, the so called whiteopaque transition) has been discovered ${ }^{18-20}$ and several laboratories have developed nice genetic tools such as gene deletion strategies, conditional expression systems and gene reporters. ${ }^{15,21-23}$ These tools have resulted in a quick advance in the basic knowledge of this fungal pathogen. The non-pathogenic yeast Saccharomyces cerevisiae has additionally served as a useful model for studying the components and functions of many genetic elements of $C$. albicans. ${ }^{24}$ It has been the case for the MAPK pathways, first identified in $S$. cerevisiae mating, and only then extended to this and other fungi. While we will emphasize for historical reasons in this review the relevance of $S$. cerevisiae as model organism, it must be stated that other fungi like Schizosaccharomyces pombe or even the pathogenic Cryptococcus neoformans may be also considered interesting comparative models for pathogenic fungi.25-27

Previous reviews from our group have highlighted the roles of MAPK pathways in $C$. albicans biology with emphasis in cell cycle regulation, ${ }^{28}$ virulence ${ }^{5,29}$ and cell wall construction. ${ }^{30,31}$ We will focus here, on the interplay between the Hogl and the Cekl-mediated pathways as they play complementary roles in this organism. Both routes influence essential aspects of fungal pathogens biology but we will 
focus here on three aspects essential for virulence: i) morphogenesis, which plays an important role in fungal virulence as a mechanism affecting the adhesion, the invasion and the dissemination of the fungus; ii) stress, which is relevant in terms of adaptation to a host-changing environment and iii) cell wall construction, which is crucial in terms of interaction of the fungi with the mammalian host and, in particular, immune cells.

\section{Overview of Saccharomyces cerevisiae mitogen-activated protein kinase pathways}

In $S$. cerevisiae, three well-characterized MAPK pathways are present: the cell integrity (CWI pathway), mediated by the Slt2 MAPK (that regulates the cell wall integrity), the high osmolarity glycerol (HOG) pathway (that responds to external increases in osmolarity) and the Kss1/Fus3 cascades (involved both in filamentous and invasive growth and mating).

The CWI pathway is activated by cell wall damage through several cellular surface proteins like Mid2, Wsc1-4 and Mtl1. ${ }^{32-34}$ Once activated, the activation of the small GTPase Roml takes place which interacts -and activates- $\mathrm{Pkc1}$, the protein kinase $\mathrm{C}$ homolog in yeast $^{35}$ triggering the signal to the MAPK module (Bck1, Mkk1/2 and Slt2). Once activated, Slt2 regulates transcriptional activation through two main TF, the SBF complex and RIm1.36,37

The HOG pathway is essential for adaptation to osmotic stress regulating the accumulation of glycerol, an intracellular compatible solute. ${ }^{38-40}$ This osmotic stress also induces a transient cell cycle arrest and inhibition of protein synthesis. ${ }^{41}$ It is composed by two upstream independent branches that activate different mitogen-activated protein kinase kinase kinases (MAPKKKs). The SLN1-branch comprises the SIn1 two-component system and the phosphorelay proteins Ypd1 and Sskl and regulates the activation of the redundant MAPKKK Ssk2/22.40,42-44 The SHO1-branch activates the MAРКKK Ste11 by the participation of the osmosensors Msb2 (a mucin type protein in yeast) and the Hkrl mucin-like transmembrane protein, ${ }^{45,46}$ the transmembrane protein Opy2,2,47,48 the GTPase Ste20 and the Ste11interacting protein Ste50.49,50 Both branches converge and activate the Pbs2 MAPKK, which in turn phosphorylates the Hog1 MAPK that regulates the appropriate transcriptional response through the TF Msn1, Hotl, Skol and Smp1.51-54

The Fus3/Kss1 pathway is involved in at least 3 processes (mating, invasive and vegetative growth). Mating is induced by the presence of pheromones which is sensed by the
Ste2, Ste3 receptors leading the activation of the MAPK module (Ste11-Ste7-Fus3/Kss1) through intermediate G-proteins (Ste4, Gpal, Ste18), the PAK Ste20, the scaffold protein Ste5 and the small protein Ste50.55 Pheromone specific gene expression is controlled by the TF Ste12. Some of the mating elements are also involved in the response to nutritional starvation inducing pseudohyphal or invasive mode of growth in diploid and haploid cells respectively, $, 5,57$ such as Ste20, Ste11 or Ste7 by a mechanism that does not involved Ste5. Transcriptional response is regulated through the shared TF Ste12 but also by Tec1.58 Upstream elements of the cascade are shared with other pathways. Shol is required for diploid pseudohyphal growth. ${ }^{59}$ Nice epistasis analysis revealed that the mucin-like protein Msb2 acts as a sensor of the pathway upstream Shol and both elements mediate the activation of Ste20.60,61 This pathway shares other elements that participate in the previously mentioned HOG cascade, which are the upstream elements Sho1 and Msb2 and 0py2 that ensure Ste11 activation through its recruitment to the plasma membrane though Ste50 and Cdc42.48,62 Elements of this route do also participate in the biogenesis of the cell wall through the so-called STE vegetative growth (SVG) pathway, ${ }^{60}$ essential when a glycosylation defect is present in the cell and promotes vegetative growth in normal (that is, nonstressed) cells. ${ }^{60,63}$

\section{Role for the Cek1 pathway in the response to osmotic and oxidative stress in C. albicans}

The HOG pathway was first described because of its role in the adaptation to increases in external osmolarity in $S$. cerevisiae. Under this stress, Hogl becomes activated in a Pbs2-dependent manner64 as occurs in C. albicans, ${ }^{65}$ and this input comes from two different upstream branches.

Initial attempts to analyze the role of the Shol-branch in C. albicans involved the identification and characterization of putative elements of the route, as many of them were functional in $S$. cerevisiae. Deletion of the SSK1 regulator does not block phosphorylation to Hog1 in response to osmotic stress. ${ }^{66}$ The transmembrane adaptor molecule Shol, that was cloned by its ability to complement the osmotic sensitivity of $S$. cerevisiae ssk1 ssk2 ssk22 mutants, is not involved in activation of Hog 167 and shol mutants are perfectly able to grow under high osmolarity (both sorbitol and sodium chloride) and transmit the stimulus to Hogl under these conditions. ${ }^{67}$ It however, plays an important role in the activation of Cek1 under a wide range on situations. These results suggested important functional differences between both organisms. It was later confirmed that CaSte11 is not involved in transmitting the signal to Hog1 upon osmotic stress and that deletion of CaSTE11 has no evident phenotype when cells are exposed to either $\mathrm{NaCl}$ or sorbitol. In contrast, deletion of SSK2 renders cells unable to grow under high concentration of $\mathrm{NaCl}$ or sorbitol and unable to activate Hog1 by phosphorylation under these conditions. ${ }^{68}$ In an attempt to identify upstream elements of the branch/route, the signaling mucin Msb2 was also cloned and epistatic analysis with $\mathrm{SHO1}$ was made. MSB2 encodes a transmembrane protein that interacts with Shol in $S$. cerevisiae and is involved in Hog1 signaling. ${ }^{61}$ Interestingly, deletion of MSB2 either alone or in combination with SHO1, had no effect on Hogl phosphorylation but it does in Cek1 activation. ${ }^{69}$

Based on these findings, it can be proposed that in $C$. albicans osmotic stress activates Hogl via exclusively the Ssk1-branch and that the Shol branch is really involved in activating and transmitting the stimulus to Cekl, not playing a role in osmoadaptation. From a molecular point of view, it is also not clear how this occurs. A recent work from our group has characterized 0py2. In S. cerevisiae, 0py2 is a membrane-anchored protein that interacts with Ste50 and is involved in Hogl activation.2,47 This is mediated by the presence of a specific region in Sc0py2 that becomes phosphorylated by the Yck1/2 kinases, allowing the interaction with Ste50.62 As expected, in $C$. albicans this protein does not participate in Hog1 signaling but rather in Cek1 signaling. ${ }^{70}$ Sequence analysis of $\mathrm{CaOpy} 2$ reveals that this protein is devoid of the Ste50-interaction phosphorylated motif, which could explain, at least partially, the situation. This interpretation still requires experimental confirmation. However, there are still certain incongruences in this scenario. Deletion of SHO1, MSB2 and even SSK1 has not effect in Hog1 phosphorylation ${ }^{69}$ as indicated; however, and most interestingly, cells are defective (almost similar to hogl mutants) in growth under high osmolarity which in turn, points towards a role for the Cek1 pathway in growth under osmotic stress pressure by a mechanism which is, apparently, independent of Hogl activation. These cells are able to activate Hogl by phosphorylation, activate a HOG1-gene reporter, and increase glycerol content (a compatible solute) upon osmotic stress. In other words, activation of Hogl is not sufficient to sustain growth when certain elements of the Cek1-pathway are missing.

The HOG pathway is also implicated in the response to other stresses, such as oxidative stress. ${ }^{71,72}$ This type of stress is, of course, more meaningful for a host-interacting microbe that is continuously exposed to the 
killing oxidative effects of macrophages at the mucosa or neutrophils during dissemination to internal organs. SSK1 deletion is essential to cope with the presence of oxidants and for transmitting the signal to Hog1 via Ssk2 (not Ste11) and Pbs2.65,66,68 In contrast, shol mutants are not defective in Hog1 phosphorylation upon this stress, albeit these mutants are slightly sensitive to oxidative stress. ${ }^{67} \mathrm{~A}$ similar situation occurs with other mutants of the pathway. ${ }^{73,74}$ The Cek1-mediated pathway, therefore, has no clear role in the survival under oxidative stress. However, an important point is that Cek1 activation is clearly altered upon oxidative stress. The addition of external oxidative compounds to actively growing cells where Cek1 is phosphorylated (activated) results in a quick dephosphorylation of this MAPK in parallel with the activation of Hogl and/or Mkc1, the cell integrity MAPK. ${ }^{75}$ Oxidative stress normally results in cell cycle arrest, as cells need all the cellular machinery to cope this danger. ${ }^{76}$ In $S$. cerevisiae, the presence of pheromone induces a cell cycle arrest through the factor arrest 1 (Far1), as it occurs in $C$. albicans. ${ }^{77,78}$ It is also demonstrated that upon stress, cells avoid the progression of the cell cycle and different MAPKs have been involved in this process. Hogl regulates cell cycle arrest upon an osmotic stress by mechanisms that involve downregulation of cyclins and stabilization of the Sicl inhibitor. ${ }^{41}$ In the case of Fus3/Kss1 MAPKs, both MAPKs induce G1 arrest prior mating by mechanisms that are partially independent on Farl and through overlapping and distinct functions. ${ }^{79}$ Therefore, oxidative mediated-activation of Cek1 could be explained if we consider Cek1 as part of the mechanism that the cells require to resume growth. It is revealing in this context that $C E K 1$ was indeed identified by a functional screening with the aim of iso- lating $C$. albicans genes able to interfere in the $S$. cerevisiae pheromone-induced cell cycle $\operatorname{arrest}^{80}$ (Table 1).

\section{The Cek1-pathway and morphogenesis}

One of the important characteristics of $C$. albicans in pathogenesis is its ability to switch between different morphologies. ${ }^{81,82}$ The yeast (unicellular) form is able, under certain conditions, to undergo transformation into hyphal cells, leading to the formation of a mycelium. Such conditions are high temperature, the presence of serum or different chemicals (like proline or $\mathrm{N}$-acetyl glucosamine) or neutral $\mathrm{pH}$. $C$. albicans can also for pseudohyphi, morphological distinct from true hyphi and chlamydospores which are thick walled structures with a largely unknown function. Morphogenesis is highly relevant in terms of pathogenicity as illustrated by several works where mutants unable to undergo the yeast-to-hypha transition are avirulent in certain animal models. ${ }^{83}$ This, however, also occurs with mutants that are permanently locked in the hyphal form ${ }^{84}$ and recent studies indicate that more than a specific morphogenetic state, it is the ability to switch between different morphologies what really favors adaptation to the host. 85 This situation makes sense, as within infection (or dissemination) yeast and hyphal forms may differ in terms of terms of penetration, avoiding phagocytosis, proliferation or dissemination.

Different lines of evidence indicate a positive role for the Cek1-pathway in regulating filamentation in $C$. albicans. Cells lacking the PAK Cst20, the MAPKK Hst7 or the TF Cph1 are defective in hyphal formation on solid agar medium. Analysis of the Cppl, a putative Cek1- phosphatase, 86 revealed that $c p p 1$ mutants are hyperinvasive, and deletion of $C E K 1$ in $c p p 1$ mutants suppresses this invasive phenotype ${ }^{87}$ suggesting this as a mechanism involved in controlling the intensity of the response via dephosphorylation of the MAPK. These studies, in any case, highlight the importance of well defining the experimental conditions used to assess morphogenetic switches in Candida. cek1 mutants are apparently normal in serum-induced liquid filamentation assays. ${ }^{88}$ A nutritional rich medium that is commonly used as test of filamentous formation. Such conditions differ, however, from solid medium, where cells are first restrained in movement and, second, nutrients may become limiting. Like Cst20, Hst7 and Cph1, Cek1 is also required for this type of agar-invasive hypha formation on different solid media such as Lee's, Spider (mannitol as carbon source) or Synthetic Low Ammonium Dextrose Histidine (nitrogen limiting conditions). ${ }^{87}$ As it happens in $S$. cerevisiae, ${ }^{48,59}$ the Shol adaptor protein and the Msb2 mucin are upstream elements of this cascade and also play a role in agar invasion during starvation. ${ }^{69}$ These results suggests that Cekl could indeed become activated under these conditions and, in fact, it has been described that Cek1 was phosphorylated, at least partially, via a membrane protein called Dfi1.89 It has been recently shown how cathecins inhibit $C$. albicans morphogenesis and this results in a decrease in hyphal specific $C E K 1$-mediated gene expression as well as Cek1 phosphorylation. ${ }^{90}$ Also, subinhibitory concentrations of hydrogen peroxide actives pseudohyphal formation in a process that seems to be also partially dependent on this MAPK pathway. 91

In close contrast with the situation with $C E K 1$, the HOG pathway plays an opposite role in $C$. albicans morphogenesis. Under non-

Table 1. Roles and stimuli related to the high osmolarity glycerol and Cek1 pathways in C. albicans.

\begin{tabular}{|c|c|c|c|}
\hline Type of stress & Role of HOG and SVG pathways & Activation of the MAPKs & Differences with S. cerevisiae \\
\hline Osmotic & $\begin{array}{l}\text { HOG pathway is essential for survival } \\
\text { SVG is dispensable for survival }\end{array}$ & $\begin{array}{l}\text { Hogl becomes phosphorylated } \\
\text { Cekl becomes desphosphorylated }\end{array}$ & $\begin{array}{l}\text { Only one MAPKKK Ssk2 } \\
\text { No apparent role for the SHO1 branch }\end{array}$ \\
\hline Oxidative & $\begin{array}{l}\text { HOG pathway is essential for survival } \\
\text { SVG is dispensable for survival }\end{array}$ & $\begin{array}{l}\text { Hogl becomes activated } \\
\text { Cek1 becomes desphosphorylated }\end{array}$ & $\begin{array}{l}\text { Only one MAPKKK Ssk2 } \\
\text { Ssk1 is essential for signaling } \\
\text { No apparent role for the SHO1 branch }\end{array}$ \\
\hline Cell wall damage & $\begin{array}{l}\text { Opposite but dependent roles: } \\
\text { SVG is essential for survival } \\
\text { SVG compensates defects in the HOG pathway }\end{array}$ & $\begin{array}{l}\text { Cek1 becomes activated } \\
\text { Cek1 is constitutively } \\
\text { phosphorylated in the absence of a } \\
\text { functional HOG pathway }\end{array}$ & $\begin{array}{l}\text { Absence of a functional HOG pathway } \\
\text { induces activation of the SVG pathway } \\
\text { even in the absence of stimuli }\end{array}$ \\
\hline
\end{tabular}

HOG, high osmolarity glycerol; SVG, STE vegetative growth; MAPKs, mitogen-activated protein kinases; MAPKKK, mitogen-activated protein kinase kinase kinase. 
inducing conditions, the absence of Hogl (MAPK) leads to enhance hyphal formation, a phenotype also observed with pbs2 mutants (MAPKK). As occurs with the hog1 mutant, cells lacking the TF SKO1 (a target of the HOG pathway in $S$. cerevisiae) also show an increased ability to form hypha under noninducing conditions. This correlates with transcriptomal analysis that indicates repression of certain hyphal related genes (such as ECE1 or $H W P 1$ ); interestingly, this repression was observed at both 30 and $37^{\circ} \mathrm{C}$, while the repression of filamentation mediated by Skol was only observed at $37^{\circ} \mathrm{C} .92 \mathrm{An}$ important difference with the situation in $S$. cerevisiae is that in $C$. albicans there is only one MAPKKK involved in this negative regulation (CaSSK2) and only Cassk2 mutants, but not Caste11 mutants, are hyperfilamentous. In agreement with this, the transcription profile of different filamentous growth-regulated targets is similar in $s s k 2$ and hog 1 mutants. ${ }^{65,68,93}$ Interestin gly, however, the repression mediated by the HOG pathway is independent on the Cek1 MAPK. This was evidenced when the hyperfilamentation phenotypes of the hog1 mutants were still present when CLA4, CST20, HST7 or CPP1 were deleted. 67,73

It must be mentioned here that certain elements of the Cek1 pathway (CST20, CLA4, $H S T 7, C E K 1, C P H 1$ or CPP1) do not affect the production of chlamydospospores, thick walled structures with unknown function in Candida. However, deletion of certain upstream elements of the Cek1 route restores the inability of hog1 mutants to form these structures. ${ }^{73}$ These data demonstrate that both routes (Cek1 and HOG) regulate in an independent and opposite manner two different morphogenetic processes in C. albicans (Figure 1).

\section{The Cek1 route as a sensor of cell wall damage and glycosylation}

The fungal cell wall is an essential structure for fungal cells that changes continuously in response to the environmental and growth conditions. Not only determinates the shape and morphology of the cell but its integrity is essential for the survival in the host. Since mammalian cells lack this structure, it is a preferred suitable target for new antifungals such as equinocandins. ${ }^{94}$ We provide here some recent findings that indicate a role for this route in modeling the cell wall.

Mutants of the SVG pathway (cek1, hst7, cst20, cla4, msb2 and shol) are susceptible to compounds that alter biogenesis of the cell wall such as Congo red, calcofluor white, equinocandins or the glucanase-enriched zymoliase. ${ }^{67,73,95}$ Defects in the cell wall, either by the addition of drugs or by specific muta- tions in cell wall genes (such as Och196), result in the activation of Cek1. Cek1 phosphorylation has been described to be mainly Sholdependent, since shol mutants blocked completely this activation under all stimuli described except for tunicamycin phosphorylation that is mainly driven through Msb2.67,74 In close contrast, the absence of SSK1, SSK2, $P B S 2$ or $H O G 1$, render cells with increased resistance to the presence in the media of these cell wall inhibitors. ${ }^{65,67,68,73,74,93}$ Mutants in the HOG pathway constitutively activate Cek1 and the absence of the Cpp1 phosphatase confers a resistant phenotype. 67,73 This phosphorylation is completely abolished when we delete any of the elements of the SVG pathway (as it occurs in shol hog1, hst7 hog1 or cek1 hog1 double mutants) becoming susceptible to the presence of these compounds. This indicates that the Congo red resistance observed in mutants lacking a functional HOG pathway is probably due to a hyperactivation of the Cek1 mediated pathway. ${ }^{73}$

The precise role of the Cek1 pathway in cell wall biogenesis is difficult to determine given the involvement of the two remaining cascades (Hogl and Mkcl-dependent) and other signaling routes in this process. For example, the calcineurin pathway participates in regulating chitin synthesis upon a cell wall stress in coordination with the HOG and PKC MAPK path- ways. 97 The PKC1-pathway, regulated by the MAPK Mkcl (homologue to ScSlt2), $, 8,99,100$ is essential for the integrity of the cell wall and becomes activated upon the addition of different cell wall inhibitors such as zymoliase, calcofluor white, tunicamycin, etc., ${ }^{75,101}$ or due to gene deletions as it occurs in $\mathrm{N}$-mannan mutants such as mns1.102 It is not surprising, also, that different morphological states differ in their cell wall and multiple hyphal specific genes have been described (as $A L S 3, A L S 8$, $H W P 1, H Y R 1$, etc...), some of them being regulated by Efg 1 at their transcriptional level.103 Multiple growth and stress conditions affect the cell wall composition and structure. The medium and growth conditions (low $\mathrm{pH}$ and temperature) and cell wall stress, due to the addition of external compounds or due to an internal mutation (as mnn9, pmt1, bgl2, phrl, etc. mutants), renders in cell wall differences.104,105 In C. albicans, a salvage mechanism similar to the one described in $S$. cerevisiae that becomes activated under cell wall stress, the so called compensatory mechanism, ${ }^{105,106}$ is expected to take place, resulting, among others, in an increased chitin level on the lateral cell wall that ensure the integrity of this structure. In addition, the role of Cek1 has been recently shown to be influenced by the Hsp90 chaperone ${ }^{107}$ or the small Hsp21,108 which in turn indicates a tight temperature-
Sensors I adaptors

Transcription factor

Kinase Response

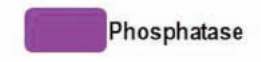

a ( 
dependent regulation of the pathway.

An important role for this pathway is emerging as sensor of glycosylation alterations. As occurs in $S$. cerevisiae, ${ }^{60}$ changes in glycosylation activate Cekl and hyperactivation of the MAPK is observed when $\mathrm{N}$ or 0-glycosylation is impaired ${ }^{96,101}$ either by mutants defective in this process or by the addition of certain chemicals. This activation is dependent, at least in the case of tunicamycin, on $\mathrm{Msb2}^{69}$ and results in the induction of some PMT genes through the transcription factor Ace2. ${ }^{109}$ In $S$. cerevisi$a e$, the cleavage and the secretion of the extracellular Msb2 N-domain by the aspartyl protease Yps1 is required for the activation of Msb2 and therefore for transmitting the signal to Kss1, with the secreted domain showing an inhibitory role. 110 In $C$. albicans, mutational analysis reveal that the transmembrane domain of Msb2 is essential for signaling to Cek1, while a specific N-proximal domain also seems to play an inhibitory role as deletion of this region results in permanent hyperactivation of the route. ${ }^{111}$ It has been recently reported that in $C$. albicans, the secreted aspartyl protease Sap8 may be the processing protease responsible for Msb2 shedding. ${ }^{112}$ Therefore, Msb2 fulfills a clear role as a sensor of wall damage that connects this stimulus via Shol with a cell wall biogenesis cascade.

Upon tunicamycin addition, Cek1 is not only activated, but there is also a boost in the amount of Cek1. This is probably related to the fact that Cek1 function is regulated both postranslationally and by proteolysis degradation through the ubiquitin proteasome degradation pathway. Moreover, Cek1 is also regulated by proteasome and it has been suggested to be a short lived protein. This was demonstrated by using a conditional proteasome mutant (prel) where Cekl, but not other MAPKs such as Hog1, was found to be faster degraded. ${ }^{74}$

While a role for the Cek1 pathway in cell wall construction seems to be clear, the precise mechanism by which this takes place is not. At the molecular level, the surface of $c e k 1$ mutants is more leaky as revealed by electron microcopy studies (Román E., 2009, unpublished data) and there are important differences in the mannan, similar to those showed by mutants in Chk1 or Sho1.113 Importantly, this results in an increased exposure of $\beta$-glucan, a major component of the cell wall.114 In yeast cells, glucan in normally hindered under the mannan layer and is not accessible to outer cells, being only accessible in bud scars. In fact, a genetic network has been recently described in $S$. cerevisiae where several signaling elements like Cla4 or Slt2 (the Mkc1 homologue) have been positioned.115 As bud scars are not present in true filaments, the switch from unicellular mode of growth to mycelium has been proposed as a morphological mechanism of escape from immune cells. Therefore, dimorphism may play a role in regu- lating the immune response. ${ }^{116}$ In fact, $c e k 1$ and hst 7 mutants show an altered $\beta$-glucan exposure; this, has functional consequences as the Dectin-1 mediated immune response is different in hDCs and transiently transfected Dectin 1 cells. This altered response could partially explain the defects in virulence associated to cek1 mutants. ${ }^{87}$ This statement may be a simplification of the real situation during infection, where several changes do take place, 117 in particular as the result of treatment with the antifungals echinocandins, inhibitors of $\beta$-glucan synthesis.

\section{Concluding remarks}

It is now clear that while similar cascades exist both in $S$. cerevisiae and $C$. albicans and most elements are found in both organisms, important differences do exist. In particular, we provide in this review evidences from different research groups that reveal the role of the Cek1 cascade in morphogenesis and the regulation of cell wall remodeling rather than participating in the adaptation to osmotic stress. Why is this occurring? One can obviously invoke evolutionary reshaping of signaling pathways. It is conceivable that in its natural environment, $S$. cerevisiae may be more prone to experience osmolarity changes; this could occur in wine/grapes and the surface of other biological material where it can be exposed to subsequent cycles of dehydration. In contrast, $C$. albicans seems to have evolved closely linked to humans, and in this host, changes in osmolarity (up to $1 \mathrm{M}$ where the phenotype of hogl is evident) are not normally encountered. It has been hypothesized that in the kidneys, increases in osmolarity are high, but this is not a common evolutionary niche for $C$. albicans, which is normally found in the gastrointestinal tract and vagina. Maybe $C$. albicans has uncoupled this branch from feeding into the HOG pathway and accommodated it in the Cekl pathway, which is a more necessary event to accomplish cell wall biogenesis during the commensal status (see below). In this context, it must be also remembered that at the top of the Cekl pathway lies a mucin that connects glycosylation defects with signaling events. However, the secreted portion of this protein is also an important defense mechanism against antimicrobial peptides such as LL-37 or histatins ${ }^{111}$ in vitro, which may play an important role in fungal infections. ${ }^{118}$ Such antimicrobial peptides are frequently encountered in habitats like the vagina and oral/gastrointestinal mucosal surfaces where $C$. albicans normally inhabits. Evolutionary pressure may have connected both functions for adaptation to the host.

Conditions encountered by pathogenic fungi are therefore different from environmental organisms. MAPK routes have adapted by evolution in the way they sense, trigger and respond to these stimuli. Knowledge of them will allow exploiting the difference that exist between fungal and mammalian routes, an aspect that can be productive in terms of antifungal research in a future.

\section{References}

1. Martin H, Flandez M, Nombela C, et al. Protein phosphatases in MAPK signaling: we keep learning from yeast. Mol Microbiol 2005;58:6-16.

2. Cote P, Sulea T, Dignard D et al. Evolutionary reshaping of fungal mating pathway scaffold proteins. MBio 2011;2:e00230-10.

3. Bahn YS, Kojima K, Cox GM, et al. A unique fungal two-component system regulates stress responses, drug sensitivity, sexual development, and virulence of Cryptococcus neoformans. Mol Biol Cell 2006;17:3122-35.

4. Heitman J. Sexual reproduction and the evolution of microbial pathogens. Curr Biol 2006;16:R711-25.

5. Román E, Arana DM, Nombela C, et al. MAP kinase pathways as regulators of fungal virulence. Trends Microbiol 2007;15:181-90.

6. Sanglard D and Odds FC. Resistance of Candida species to antifungal agents: molecular mechanisms and clinical consequences. Lancet Infect Dis 2002;2:73-85.

7. Alangaden GJ. Nosocomial fungal infections: epidemiology, infection control, and prevention. Infect Dis Clin North Am 2011;25:201-25.

8. Pfaller MA, Diekema DJ. Epidemiology of invasive candidiasis: a persistent public health problem. Clin Microbiol Rev 2007; 20:133-63.

9. Richardson MD. Changing patterns and trends in systemic fungal infections. $\mathrm{J}$ Antimicrob Chemother 2005;56:i5-11.

10. Wisplinghoff H, Bischoff T, Tallent SM, et al. Nosocomial bloodstream infections in US hospitals: analysis of 24,179 cases from a prospective nationwide surveillance study. Clin Infect Dis 2004;39:309-17.

11. Hernandez-Santos N, Gaffen SL. Th17 cells in immunity to Candida albicans. Cell Host Microbe 2012;11:425-35.

12. Gaffen SL, Hernandez-Santos N, Peterson AC. IL-17 signaling in host defense against Candida albicans. Immunol Res 2011;50:181-7.

13. van de Veerdonk FL, Kullberg BJ, Netea MG. Pathogenesis of invasive candidiasis. Curr Opin Crit Care 2010;16:453-9. 
14. Romani L. Immunity to fungal infections. Nat Rev Immunol 2004;4:1-23.

15. De Backer MD, Magee PT, Pla J. Recent developments in molecular genetics of Candida albicans. Annu Rev Microbiol 2000;54:463-98.

16. Pla J, Gil C, Monteoliva L, et al. Understanding Candida albicans at the molecular level. Yeast 1996;12:1677-702.

17. Braun BR, Van Het HM, d'Enfert C, et al. A human-curated annotation of the Candida albicans genome. PLoS Genet 2005; 1:e1.

18. Bennett RJ, Johnson AD. Mating in Candida albicans and the search for a sexual cycle. Annu Rev Microbiol 2005;59:233-55.

19. Hickman MA, Zeng G, Forche A, et al. The 'obligate diploid' Candida albicans forms mating-competent haploids. Nature 2013;494:55-9.

20. Forche A, Alby K, Schaefer D, et al. The parasexual cycle in Candida albicans provides an alternative pathway to meiosis for the formation of recombinant strains. PLoS Biol 2008;6:e110.

21. Hernday AD, Noble SM, Mitrovich QM, et al. Genetics and molecular biology in Candida albicans. Methods Enzymol 2010;470:737-58.

22. Noble SM and Johnson AD. Genetics of Candida albicans, a diploid human fungal pathogen. Annu.Rev.Genet 2007; 41:193-211.

23. Morschhauser J, Staib P, Kohler G. Targeted gene deletion in Candida albicans wild-type strains by MPAR flipping. Methods Mol Med 2005;118:35-44.

24. Berman J, Sudbery PE. Candida albicans: a molecular revolution built on lessons from budding yeast. Nat Rev Genet 2002; 3:918-30.

25. Perez P, Cansado J. Cell integrity signaling and response to stress in fission yeast. Curr Protein Pept Sci 2010;11:680-92.

26. Ikner A, Shiozaki K. Yeast signaling pathways in the oxidative stress response. Mutat Res 2005;569:13-27.

27. Idnurm A, Bahn YS, Nielsen $K$, et al. Deciphering the model pathogenic fungus Cryptococcus neoformans. Nat Rev Microbiol 2005;3:753-64.

28. Correia I, Alonso-Monge R, Pla J. MAPK cell-cycle regulation in Saccharomyces cerevisiae and Candida albicans. Future Microbiol 2010;5:1125-41.

29. Alonso-Monge R, Román E, Pla J, Nombela $C$. A host view of the fungal cell wall. In: Baquero Mochales F, Nombela C, Cassel GH, Gutiérrez-Fuentes JA, eds. Evolutionary biology of bacterial and fungal pathogens. Washington D.C.: ASM Press; 2009. pp 105-115.

30. Navarro-García F, Eisman B, Román E, et al. Signal transduction pathways and cellwall construction in Candida albicans. Med Mycol 2001;39:87-100.

31. Ernst JF, Pla J. Signaling the glycoshield: maintenance of the Candida albicans cell wall. Int J Med Microbiol 2011;301:378-83.

32. Verna J, Lodder A, Lee K, et al. A family of genes required for maintenance of cell wall integrity and for the stress response in Saccharomyces cerevisiae. Proc Natl Acad Sci U S A 1997;94:13804-9.

33. Ketela T, Green R, Bussey $H$. Saccharomyces cerevisiae Mid2p is a potential cell wall stress sensor and upstream activator of the PKC1-MPK1 cell integrity pathway. J Bacteriol 1999;181: 3330-40.

34. Rajavel M, Philip B, Buehrer BM, et al. Mid2 is a putative sensor for cell integrity signaling in Saccharomyces cerevisiae. Mol Cell Biol 1999;19:3969-76.

35. Levin DE. Cell wall integrity signaling in Saccharomyces cerevisiae. Microbiol Mol Biol Rev 2005;69:262-91.

36. Watanabe Y, Takaesu G, Hagiwara M, et al. Characterization of a serum response factor-like protein in Saccharomyces cerevisiae, Rlm1, which has transcriptional activity regulated by the Mpk1 (Slt2) mitogen-activated protein kinase pathway. Mol Cell Biol 1997;17:2615-23.

37. Baetz K, Moffat J, Haynes J, et al. Transcriptional coregulation by the cell integrity mitogen-activated protein kinase Slt2 and the cell cycle regulator Swi4. Mol Cell Biol 2001;21:6515-28.

38. Saito H, Tatebayashi K. Regulation of the osmoregulatory HOG MAPK cascade in yeast. J Biochem (Tokyo) 2004;136:267-72.

39. Posas F, Takekawa M, Saito H. Signal transduction by MAP kinase cascades in budding yeast. Curr Opin Microbiol 1998;1:175-82.

40. Hohmann S. Osmotic stress signaling and osmoadaptation in yeasts. Microbiol Mol Biol Rev 2002;66:300-72.

41. Escote X, Zapater M, Clotet J, et al. Hog1 mediates cell-cycle arrest in G1 phase by the dual targeting of Sicl. Nat Cell Biol 2004;6:997-1002.

42. Posas F, Saito H. Activation of the yeast SSK2 MAP kinase kinase kinase by the SSK1 two- component response regulator. EMBO J 1998;17:1385-94.

43. de Nadal E, Alepuz PM, Posas F. Dealing with osmostress through MAP kinase activation. EMBO Rep 2002;3:735-40.

44. Reiser V, Raitt DC, Saito H. Yeast osmosensor Sln1 and plant cytokinin receptor Crel respond to changes in turgor pressure. J Cell Biol 2003;161:1035-40.

45. O'Rourke SM, Herskowitz I. A third osmosensing branch in Saccharomyces cerevisiae requires the Msb2 protein and functions in parallel with the Shol branch. Mol Cell Biol 2002;22:4739-49.

46. Tatebayashi K, Tanaka K, Yang HY, et al. Transmembrane mucins Hkrl and Msb2 are putative osmosensors in the SHO1 branch of yeast HOG pathway. EMBO J 2007;26:3521-33.

47. Wu C, Jansen G, Zhang J, et al. Adaptor protein Ste50p links the Ste11p MEKK to the HOG pathway through plasma membrane association. Genes Dev 2006;20: 734-46.

48. Pitoniak A, Birkaya B, Dionne HS, et al. The signaling mucins Msb2 and Hkr1 differentially regulate the filamentation MAPK pathway and contribute to a multimodal response. Mol Biol Cell 2009;20: 3101-14.

49. Maeda T, Takekawa M, Saito H. Activation of yeast PBS2 MAPKK by MAPKKKs or by binding of an SH3-containing osmosensor. Science 1995;269:554-58X.

50. Raitt DC, Posas F, Saito H. Yeast Cdc42 GTPase and Ste20 PAK-like kinase regulate Sho1-dependent activation of the Hog1 MAPK pathway. EMBO J 2000;19: 4623-31.

51. Rep M, Reiser V, Gartner U, et al. Osmotic stress-induced gene expression in Saccharomyces cerevisiae requires Msn1p and the novel nuclear factor Hotlp. Mol Cell Biol 1999;19:5474-85.

52. Rep M, Proft M, Remize F, et al. The Saccharomyces cerevisiae Skolp transcription factor mediates HOG pathwaydependent osmotic regulation of a set of genes encoding enzymes implicated in protection from oxidative damage. Mol Microbiol 2001;40:1067-83.

53. Proft M, Gibbons FD, Copeland M, et al Genomewide identification of Skol target promoters reveals a regulatory network that operates in response to osmotic stress in Saccharomyces cerevisiae. Eukaryot Cell 2005;4:1343-52.

54. Proft M, Serrano R. Repressors and upstream repressing sequences of the stress-regulated ENA1 gene in Saccharomyces cerevisiae: bZIP protein Skolp confers HOG- dependent osmotic regulation. Mol Cell Biol 1999;19:537-46.

55. Elion EA. Pheromone response, mating and cell biology. Curr Opin Microbiol 2000;3:573-58.

56. Gimeno CJ, Ljungdahl PO, Styles CA, et al. Unipolar cell divisions in the yeast $\mathrm{S}$. cerevisiae lead to filamentous growth: regulation by starvation and RAS. Cell 1992:68:1077-90.

57. Palecek SP, Parikh AS, Kron SJ. Sensing, signaling and integrating physical processes during Saccharomyces cerevisiae invasive and filamentous growth. Microbiol 2002;148:893-907. 
58. Schweizer A, Rupp S, Taylor BN, et al. The TEA/ATTS transcription factor CaTeclp regulates hyphal development and virulence in Candida albicans. Mol Microbiol 2000;38:435-45.

59. O'Rourke SM, Herskowitz I. The Hog1 MAPK prevents cross talk between the HOG and pheromone response MAPK pathways in Saccharomyces cerevisiae. Genes Dev 1998;12:2874-86.

60. Lee BN, Elion EA. The MAPKKK Ste11 regulates vegetative growth through a kinase cascade of shared signaling components. Proc Natl Acad Sci U S A 1999;96: 12679-84.

61. Cullen PJ, Sabbagh W Jr, Graham E, et al. A signaling mucin at the head of the Cdc42- and MAPK-dependent filamentous growth pathway in yeast. Genes Dev 2004;18:1695-708.

62. Yamamoto K, Tatebayashi K, Tanaka K, et al. Dynamic control of yeast MAP kinase network by induced association and dissociation between the Ste50 scaffold and the 0py2 membrane anchor. Mol Cell 2010;40:87-98

63. Cullen PJ, Schultz J, Horecka J, et al. Defects in protein glycosylation cause SH01-dependent activation of a STE12 signaling pathway in yeast. Genetics 2000;155:1005-18.

64. Brewster JL, de Valoir T, Dwyer ND, et al. An osmosensing signal transduction pathway in yeast. Science 1993;259: 1760-3.

65. Arana DM, Nombela C, Alonso-Monge R, et al. The Pbs2 MAP kinase kinase is essential for the oxidative-stress response in the fungal pathogen Candida albicans. Microbiol 2005;151:1033-49.

66. Chauhan N, Inglis D, Román E, et al. Candida albicans response regulator gene SSK1 regulates a subset of genes whose functions are associated with cell wall biosynthesis and adaptation to oxidative stress. Eukaryot Cell 2003;2:1018-24.

67. Román E, Nombela C, Pla J. The Shol adaptor protein links oxidative stress to morphogenesis and cell wall biosynthesis in the fungal pathogen Candida albicans. Mol Cell Biol 2005;25:10611-27.

68. Cheetham J, Smith DA, da Silva DA, et al. A Single MAPKKK Regulates the Hog1 MAPK Pathway in the Pathogenic Fungus Candida albicans. Mol Biol Cell 2007;18: 4603-14.

69. Román E, Cottier F, Ernst JF et al. Msb2 signaling mucin controls activation of Cek1 mitogen-activated protein kinase in Candida albicans. Eukaryot Cell 2009;8:1235-49.

70. Herrero de DC, Roman E, Diez C, et al. The transmembrane protein 0py2 mediates activation of the Cek1 MAP kinase in
Candida albicans. Fungal Genet Biol 2013;50:21-32.

71. Alonso-Monge R, Navarro-García F, Román E, et al. The Hog1 mitogen-activated protein kinase is essential in the oxidative stress response and chlamydospore formation in Candida albicans. Eukaryot Cell 2003;2:351-61.

72. Arana DM, Alonso-Monge $\mathrm{R}, \mathrm{Du} \mathrm{C}$ et al. Differential susceptibility of mitogenactivated protein kinase pathway mutants to oxidative-mediated killing by phagocytes in the fungal pathogen Candida albicans. Cell Microbiol 2007;9:1647-59.

73. Eisman B, Alonso-Monge R, Román E, et al. The Cek1 and Hog1 mitogen-activated protein kinases play complementary roles in cell wall biogenesis and chlamydospore formation in the fungal pathogen Candida albicans. Eukaryot Cell 2006; 5:347-58.

74. Román E, Alonso-Monge R, Gong Q, et al. The Cekl MAPK is a short-lived protein regulated by quorum sensing in the fungal pathogen Candida albicans. FEMS Yeast Res 2009;9:942-55.

75. Navarro-Garcia F, Eisman B, Fiuza SM, et al. The MAP kinase Mkclp is activated under different stress conditions in Candida albicans. Microbiol 2005;151: 2737-49.

76. Wanke V, Accorsi K, Porro D, et al. In budding yeast, reactive oxygen species induce both RAS-dependent and RASindependent cell cycle-specific arrest. Mol Microbiol 1999;32:753-64.

77. Cote P, Whiteway M. The role of Candida albicans FAR1 in regulation of pheromone-mediated mating, gene expression and cell cycle arrest. Mol Microbiol 2008;68:392-404.

78. Chang F, Herskowitz I. Phosphorylation of FAR1 in response to a-Factor: a possible requirement for cell-cycle arrest. Mol Biol.Cell 1992;3:445-50.

79. Cherkasova V, Lyons DM, Elion EA. Fus3p and Ksslp control G1 arrest in Saccharomyces cerevisiae through a balance of distinct arrest and proliferative functions that operate in parallel with Farlp. Genetics 1999;151:989-1004.

80. Whiteway M, Dignard D, Thomas DY. Dominant negative selection of heterologous genes: isolation of Candida albicans genes that interfere with Saccharomyces cerevisiae mating factor-induced cell cycle arrest. Proc Natl Acad Sci U S A 1992;89:9410-4.

81. Bockmuhl DP, Krishnamurthy S, Gerads $\mathrm{M}$, et al. Distinct and redundant roles of the two protein kinase A isoforms Tpklp and Tpk2p in morphogenesis and growth of Candida albicans. Mol Microbiol 2001;42:1243-57.
82. Calderone RA, Gow NA. Host recognition by Candida species. In: Calderone RA, (ed). Candida and Candidiosis. Washington D.C.: ASM Press; 2002. pp $67-$ 86.

83. Lo HJ, Kohler JR, DiDomenico B, et al. Nonfilamentous C. albicans mutants are avirulent. Cell 1997;90:939-49.

84. Braun BR, Johnson AD. Control of filament formation in Candida albicans by the transcriptional repressor TUP1. Science 1997;277:105-9.

85. Saville SP, Lazzell AL, Monteagudo C, et al. Engineered control of cell morphology in vivo reveals distinct roles for yeast and filamentous forms of Candida albicans during infection. Eukaryot Cell 2003;2: 1053-60.

86. Guhad FA, Csank C, Jensen HE, et al. Reduced pathogenicity of a Candida albicans MAP kinase phosphatase (CPP1) mutant in the murine mastitis model. APMIS 1998;106:1049-55.

87. Csank C, Schröppel K, Leberer E, et al. Roles of the Candida albicans mitogenactivated protein kinase homolog, Cek1p, in hyphal development and systemic candidiasis. Infect Immun 1998;66:2713-21.

88. Leberer E, Harcus D, Broadbent ID, et al. Signal transduction through homologs of the Ste20p and Ste7p protein kinases can trigger hyphal formation in the pathogenic fungus Candida albicans. Proc Natl Acad Sci USA 1996;93:13217-22.

89. Zucchi PC, Davis TR, Kumamoto CA. A Candida albicans cell wall-linked protein promotes invasive filamentation into semi-solid medium. Mol Microbiol 2010; 76:733-48.

90. Saito H, Tamura M, Imai K, et al. Catechin inhibits Candida albicans dimorphism by disrupting Cek1 phosphorylation and cAMP synthesis. Microb Pathog 2013;56: 16-20.

91. Srinivasa K, Kim J, Yee $\mathrm{S}$, et al. A MAP kinase pathway is implicated in the pseudohyphal induction by hydrogen peroxide in Candica albicans. Mol Cells 2012;33:183-93.

92. Alonso-Monge R, Roman E, Arana DM, et al. The Skol protein represses the yeastto-hypha transition and regulates the oxidative stress response in Candida albicans. Fungal Genet Biol 2010;47:587-601.

93. Alonso-Monge R, Navarro-García F, Molero G, et al. Role of the mitogen-activated protein kinase Hoglp in morphogenesis and virulence of Candida albicans. J Bacteriol 1999;181:3058-68.

94. Denning DW. Echinocandin antifungal drugs. Lancet 2003;362:1142-51.

95. Arana DM, Prieto D, Roman E, et al. The role of the cell wall in fungal pathogenesis. Microb Biotechnol 2009;2:308-20. 
96. Bates S, Hughes HB, Munro CA, et al. Outer chain $\mathrm{N}$-glycans are required for cell wall integrity and virulence of Candida albicans. J Biol Chem 2006;281: 90-8.

97. Munro CA, Selvaggini S, de B I, et al. The PKC, HOG and Ca2+ signaling pathways co-ordinately regulate chitin synthesis in Candida albicans. Mol Microbiol 2007;63: 1399-413.

98. Martín H, Arroyo J, Sánchez M, et al. Activity of the yeast MAP kinase homologue Slt2 is critically required for cell integrity at 37 degrees C. Mol Gen Genet 1993;241:177-84.

99. Navarro-García F, Sanchez M, Pla J, et al. Functional characterization of the MKC1 gene of Candida albicans, which encodes a mitogen-activated protein kinase homolog related to cell integrity. Mol Cell Biol 1995;15:2197-206.

100. Navarro-García F, Alonso-Monge R, Rico $\mathrm{H}$, et al. A role for the MAP kinase gene MKC1 in cell wall construction and morphological transitions in Candida albicans. Microbiol 1998;144:411-24.

101. Cantero P, Lengsfeld C, Subanovic M, et al. Transcriptional and physiological adaptation to defective protein-0-mannosylation in Candida albicans. Mol Microbiol 2007;64:1115-28.

102. Mora-Montes HM, Bates S, Netea MG, et al. Endoplasmic reticulum alpha-glycosidases of Candida albicans are required for $\mathrm{N}$ glycosylation, cell wall integrity, and normal host-fungus interaction. Eukaryot Cell 2007;6:2184-93.
103. Leng P, Lee PR, Wu H, et al. Efgl, a morphogenetic regulator in Candida albicans, is a sequence-specific DNA binding protein. J Bacteriol 2001;183:4090-3.

104. Klis FM, De GP, Hellingwerf K. Molecular organization of the cell wall of Candida albicans. Med Mycol 2001;39:1-8.

105. Popolo L, Gualtieri T, Ragni E. The yeast cell-wall salvage pathway. Med Mycol 2001;39:111-21.

106. Smits GJ, Kapteyn JC, van den EH, et al. Cell wall dynamics in yeast. Curr Opin Microbiol 1999;2:348-52.

107. Leach MD, Budge S, Walker L, et al. Hsp90 orchestrates transcriptional regulation by Hsfl and cell wall remodelling by MAPK signaling during thermal adaptation in a pathogenic yeast. PLoS Pathog 2012;8: e1003069.

108. Mayer FL, Wilson D, Jacobsen ID, et al. Small but crucial: the novel small heat shock protein Hsp21 mediates stress adaptation and virulence in Candida albicans. PLoS ONE 2012;7:e38584.

109. Cantero PD, Ernst JF. Damage to the glycoshield activates PMT-directed 0-mannosylation via the Msb2-Cek1 pathway in Candida albicans. Mol Microbiol 2011;80: 715-25.

110. Vadaie N, Dionne H, Akajagbor DS, et al. Cleavage of the signaling mucin Msb2 by the aspartyl protease Yps1 is required for MAPK activation in yeast. J Cell Biol 2008; 181:1073-81.

111. Szafranski-Schneider E, Swidergall M, Cottier F, et al. Msb2 shedding protects
Candida albicans against antimicrobial peptides. PLoS Pathog 2012;8:e1002501.

112. Puri S, Kumar R, Chadha S, et al. Secreted aspartic protease cleavage of Candida albicans Msb2 activates Cek1 MAPK signaling affecting biofilm formation and oropharyngeal candidiasis. PLoS ONE 2012;7:e46020.

113. Li D, Williams D, Lowman D, et al. The Candida albicans histidine kinase Chklp: signaling and cell wall mannan. Fungal Genet Biol 2009;46:731-41.

114. Galan-Diez M, Arana DM, Serrano-Gomez $\mathrm{D}$, et al. Candida albicans beta-glucan exposure is controlled by the fungal CEK1-mediated mitogen-activated protein kinase pathway that modulates immune responses triggered through dectin-1. Infect.Immun 2010;78:1426-36.

115. Wheeler RT, Fink GR. A drug-sensitive genetic network masks fungi from the immune system. PLoS Pathog 2006;2:e35.

116. Gantner BN, Simmons RM, Underhill DM. Dectin-1 mediates macrophage recognition of Candida albicans yeast but not filaments. EMBO J 2005;24:1277-86.

117. Wheeler RT, Kombe D, Agarwala SD, et al. Dynamic, morphotype-specific Candida albicans beta-glucan exposure during infection and drug treatment. PLoS Pathog 2008;4:e1000227.

118. Muniz LR, Knosp C, Yeretssian G. Intestinal antimicrobial peptides during homeostasis, infection, and disease. Front Immunol 2012;3:310. 